\title{
Smart Grid Internet of Things
}

\author{
Zubair Md. Fadlullah $^{1} \cdot$ Al-Sakib Khan Pathan ${ }^{2} \cdot$ Karan Singh $^{3}$
}

Published online: 26 October 2017

(C) Springer Science+Business Media, LLC 2017

This special issue marks an important milestone combining two disciplines, namely smart grid and the Internet-of-Things (IoT). Special thanks go to the members of the editorial board, reviewers, and journal staffs, who provided invaluable support throughout this long process. A total of nine papers have been accepted in the special issue. In addition to papers accepted through open call, the special issue includes selected papers presented at the 1st EAI International Conference on Smart Grid Assisted Internet of Things (SGIoT 2017) held in Sault Ste. Marie, Ontario, Canada, during July 11-12, 2017. We would like to provide a brief overview of the selected papers that will show the variety of topics accommodated in this special issue.

First, K. Sohraby et al.'s work entitled "A Review of Wireless and Satellite-based M2M/IoT Services in Support of Smart Grids" familiarizes the readers with a survey on the state-of-the-art machine-to-machine and IoT services to facilitate smart grid communications. In addition to short-range wireless communications, the paper discusses how key

Al-Sakib Khan Pathan

spathan@ieee.org

Zubair Md. Fadlullah

zfadlullah@ieee.org

Karan Singh

karan@mail.jnu.ac.in

1 Graduate School of Information Sciences, Tohoku University, Aramaki-Aoba 6-3-09, Aoba-ku, Sendai, Miyagi

Prefecture 980-8579, Japan

2 CSE Department, Southeast University, A R Tower, 24, Kemal Ataturk Avenue, Dhaka 1213, Bangladesh

3 School of Computer \& Systems Sciences, Jawaharlal Nehru University, New Delhi, India satellite-based communication technologies such as GEO (Geostationary Orbit), LEO (Low Earth Orbit), FSS (FixedSatellite Service), HTS (High Throughput Satellite), and MSS (Mobile Satellite Service) can be exploited for IoT-enabled smart grid.

Next, "A Deferrable Energy Scheduling Algorithm in Smart Grid Distribution" by J. Gao et al. considers a realtime demand response system with an aim to solve the total cost minimization problem of real-time pricing in smart grid, particularly with an emphasis on the neighborhood area networks. The paper treats the smart grid as the Internet of Energy (IoE), a subset of the state-of-the-art IoT whereby their proposed energy scheduling algorithm is treated as an algorithm for scheduling the IoT "things" (i.e., smart meters, sensors/home appliances, and so forth).

The paper "Performance Evaluation of Large-scale $R F$ Mesh Networks in a Smart City context" from F. Malandra and B. Sanso discusses why it is essential to study the performance of large scale communication systems, particularly from the point of view of a power utility company so as to assess the feasibility of smart grid applications. A computationally efficient simulation tool is presented in this vein, which may be an excellent way for readers/future researchers to evaluate the performance of large-scale wireless mesh communication networks within the context of a smart grid Advanced Metering Infrastructure (AMI) system.

On the other hand, the work conducted by M. Zourob and R. Rao in "Hybrid Lower-Complexity Wiener Filter for PilotBased Channel Estimation for C-RS in LTE-A DL System" uses Long Term Evolution Advanced (LTE-A) communication networks by arguing that a cellular communication network may be adequate for a smart grid system deployment. This paper presents a pilot-based lower complexity wireless channel estimation for Cell-specific Reference Signals (C-RS) in the LTE-A network that significantly reduces 
computational burden. The readers can be benefitted from this paper as it addresses a critical multi-disciplinary research issue - in the IoT delivery networks, in the smart grid communication networks, and needless to say in cellular communications.

The special issue has accommodated two (2) green, energyfriendly networking solutions in the context of IoT-enabled smart grids. The paper "Modelling Green Femtocells in Smart-grids" by F. Al-Turjman demonstrates how mobile Femto Base Stations (FBSs) can play a critical role in sustaining IoT setups such as green smart grids under increasing traffic loads. On the other hand, the paper entitled "Energy Harvesting Cognitive Radio Networking for IoT-enabled Smart Grid" by M. Ozger et al. considers an entirely new networking approach by adopting energy harvesting cognitive radio technology for IoT-enabled smart grids. The aim of this paper is to exploit cognitive radio to overcome harsh channel conditions and spectrum scarcity problems in the smart grid communication network.

Two (2) security papers have been accommodated in the special issue also. The paper "A Smart Meter and Smart House Integrated to an IdM and Key-based Scheme for Providing Integral Security for a Smart Grid ICT" by V. Aberu et al. presents an integral solution for securing communication between smart meter/smart home and the utility ICT infrastructure. By avoiding hardcoded crypto-keys, the paper proposes a unique technique to address a key limitation of the state-of-the-art AMI communication. On the other hand, the paper "Hybrid Cryptography Algorithm with Precomputation for Advanced Metering Infrastructure Networks" by S. Khasawneh and M. Kadoch envisages a hybrid encryption scheme that incorporates symmetric and public key encryption to exploit the strengths of either technique. Combining such works in a broader context are critical as they can be part of the means to thwart cyber threats such as false data injection and replay attacks.

Finally, Electric Vehicles (EVs) are another important research area in micro smart grid, and the special issue does accommodate a paper in this area entitled "IoT enabled Monitoring of an Optimized Electric Vehicle's Battery System" by A. Mohammad et al. An optimization model to maximize the trade revenue for an aggregator of EVs is envisioned in this paper that emphasizes on durability of the EV battery. Furthermore, a real-time battery monitoring system using a low computation communication protocol is presented in this paper.

The works conducted by the authors in this special issue clearly indicates that there are various open issues in the route towards achieving pragmatic IoT-enabled smart grids. However, it is hoped that the special issue can provide a small yet firm step toward that direction.

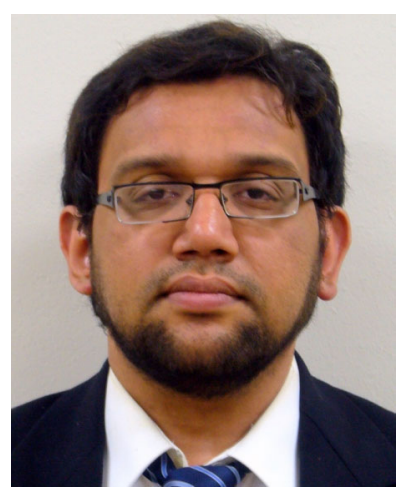

Zubair Md. Fadlullah is serving as an Associate Professor at the Graduate School of Information Sciences, Tohoku University. His research interests are in the areas of $5 \mathrm{G}$, deep learning, smart grid, network security, intrusion detection, game theory, and quality of security service provisioning mechanism. He received the Dean's award and the President's award from Tohoku University in 2011, IEEE Asia Pacific Outstanding Researcher Award in 2015, NEC foundation prize for research contributions in 2016, and several best paper awards in ICC, Globecom and IWCMC conferences. He is also editor of several journals and transactions. Email: zfadlullah@ieee.org

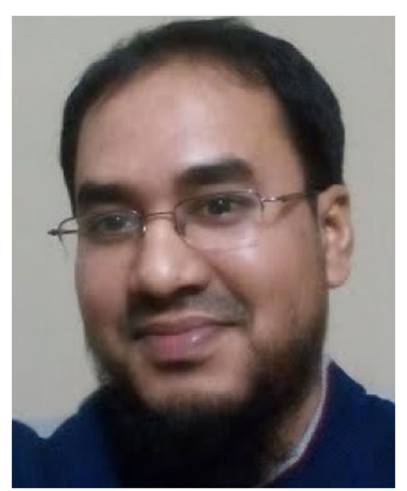

Al-Sakib Khan Pathan received $\mathrm{Ph} . \mathrm{D}$. in Computer Engineering from Kyung Hee University, South Korea and B.Sc. in Computer Science and Information Technology from Is lamic University of Technology (IUT), Bangladesh. He has several notable publications in the areas of networking, security, cloud, wireless technologies, and some multidisciplinary issues, some of which earned him awards and recognitions. He is currently an Associate Professor of CSE department at Southeast University, Bangladesh. He is serving as the Editor-in-Chief/Editor/Guest Editor of several renowned journals as well as a Chair and Committee Member in some prestigious conferences. He is a Senior Member of IEEE. Email: spathan@ieee.org

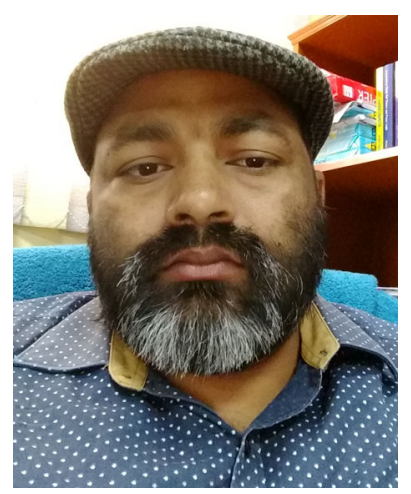

Karan Singh received the Engineering degree (Computer Science \& Engineering) from Kamala Nehru Institute of Technology, India and the M.Tech (Computer Science \& Engineering) from Motilal Nehru National Institute of Technology, India. He got Ph.D. (Computer Science \& Engineering) from MNNIT Allahabad deemed university. Currently, he is working with the School of Computer \& Systems Sciences, Jawaharlal Nehru University, New Delhi, India. His primary research interests are in computer network, computer network security, multicast communication and software defined network. He is an editor and reviewer of various prestigious journals. Email:karan@mail.jnu.ac.in 